\title{
Cuidados de transição: análise do conceito na gestão da alta hospitalar
}

\author{
Transitional care: analysis of the concept in hospital discharge management \\ Atención transicional: análisis de concepto en gestión de alta hospitalaria

\section{RESUMO}

Elizabeth Bernardino ${ }^{1}$ (1) Solange Meira de Sousa ${ }^{1}$ (D) Jaqueline Dias do Nascimento ${ }^{1}$ (D) Maria Ribeiro Lacerda ${ }^{1}$ (D) Danelia Gomez Torres ${ }^{2}$ (1) Luciana Schleder Gonçalves ${ }^{1}$ (B)

1. Universidade Federal do Paraná, Programa de Pós-graduação em Enfermagem. Curitiba, PR, Brasil.

2. Universidad Autonoma Del Estado de México, Facultad de Enfermeria y Obstetricia. Toluca de Lerdo, México.
Autor correspondente:

Solange Meira de Sousa.

E-mail: solangesmj@gmail.com

\section{Recebido em 04/11/2020.}

Aprovado em 17/08/2021.

DOl:https://doi.org/10.1590/2177-9465-EAN-2020-0435
Objetivo: analisar o conceito Cuidados de Transição no contexto da gestão da alta hospitalar. Método: reflexão analítica utilizandose a análise conceitual de Walker e Avant: seleção do conceito; definição do objetivo, identificação do uso do conceito (busca na literatura e dicionários entre setembro-dezembro/2019 nas bases de dados: PubMed, Biblioteca Virtual em Saúde e SCOPUS Consideraram-se 77 artigos que contemplaram conceito e outros termos que corroboraram o estudo; para fins metodológicos, 12 estudos possibilitaram a análise); definição dos atributos; descrição de caso modelo; descrição de casos adicionais; definição de antecedentes e consequentes; definição de indicadores empíricos. Resultados: os antecedentes cuidados fragmentados e reinternação são comuns ao conceito. Identificaram-se atributos cuidado integrado, colaboração profissional, coordenação, planejamento da alta, comunicação, integração profissional e gerenciamento de casos. Conclusão e implicações para a prática: foi oportuno analisar o conceito em conjunto com termos relacionados ao contexto da alta hospitalar. Cuidados de Transição são práticas coordenadas e eficazes para a Continuidade dos Cuidados na transferência do usuário na alta hospitalar; nesse contexto, as Enfermeiras de Ligação são potenciais protagonistas para estar à frente nesse processo de Integração. As especificidades do conceito poderão favorecer a sua compreensão e a construção de conhecimentos que repercutam no cuidado coordenado e contínuo.

Palavras-chave: Alta Hospitalar. Cuidados de Transição. Continuidade da Assistência ao Paciente. Integração dos Serviços de Saúde. Enfermeiras e Enfermeiros.

\begin{abstract}
Objective: to analyze the concept of Transitional Care in the context of hospital discharge management. Method: analytical reflection using the Walker and Avant's conceptual analysis: concept selection; definition of the objective, identification of the use of the concept (literature search and dictionaries between September-December/2019 in the databases: PubMed, Virtual Health Library and SCOPUS. A total of 77 articles that contemplated the concept and other terms that corroborated the study were considered; for methodological purposes, 12 studies enabled the analysis); definition of attributes; description of model case; description of additional cases; definition of antecedents and consequents; definition of empirical indicators. Results: the fragmented care and readmission antecedents are common to the concept. The attributes integrated care, professional collaboration, coordination, discharge planning, communication, professional integration, and case management were identified. Conclusion and Implications for practice: it was opportune to analyze the concept together with terms related to the context of hospital discharge. Transitional Care is coordinated and effective practices for the Continuity of Care in the transference of the user at hospital discharge; in this context, Liaison Nurses are potential protagonists to be ahead in this Integration process. The specificities of the concept may favor its understanding and the construction of knowledge that has repercussions on coordinated and continuous care.
\end{abstract}

Keywords: Patient Discharge; Transitional Care; Continuity of Patient Care; Intersectoral Collaboration; Nurses.

\section{RESUMEN}

Objetivo: analizar el concepto de Atención Transicional en el contexto de la gestión del alta hospitalaria. Método: reflexión analítica utilizando el análisis conceptual de Walker y Avant: selección de conceptos; definición del objetivo, identificación del uso del concepto (búsqueda en la literatura y diccionarios entre septiembre-diciembre / 2019 en las bases de datos: PubMed, Virtual Health Library y SCOPUS. Se consideraron 77 artículos que contemplaban el concepto y otros términos que corroboraban el estudio; a efectos metodológicos, 12 estudios permitieron el análisis); definición de atributos; descripción del caso modelo; descripción de casos adicionales; definición de antecedentes y consecuencias; definición de indicadores empíricos. Resultados: la atención fragmentada y los antecedentes de readmisión son comunes al concepto. Se identificaron los atributos atención integral, colaboración profesional, coordinación, planificación del alta, comunicación, integración profesional y manejo de casos Conclusión e implicaciones para la práctica: fue apropiado analizar el concepto junto con términos relacionados con el contexto del alta hospitalaria. Transition Care son prácticas coordinadas y efectivas para la Continuidad de la Atención en el traslado del usuario al alta hospitalaria; en este contexto, las Enfermeras de Enlace son potenciales protagonistas para estar a la vanguardia de este proceso de integración. La especificidad del concepto puede favorecer su comprensión y la construcción de conocimientos que inciden en la atención coordinada y continuada.

Palabras clave: Alta del Paciente; Cuidado de Transición; Continuidad de la Atención al Paciente; Colaboración Intersectorial; Enfermeras y Enfermeros. 


\section{INTRODUÇÃO}

Conceitos são expressos por meio da linguagem (termos ou palavras), permitindo a comunicação das ideias e classificando as experiências de maneira significativa ${ }^{1}$. Podem ser considerados concretos ou abstratos, possibilitando a descrição de qualquer situação². Estabelecidos ao longo do tempo por determinadas circunstâncias, os conceitos permitem expandir o conhecimento. $\mathrm{Na}$ Enfermagem, são imprescindíveis para que o cuidado prestado possa ser executado com qualidade e, considerando os vários contextos, sejam visualizados de maneira a não gerar ambiguidade na assistência ${ }^{3-7}$.

A necessidade de hospitalização é determinada, principalmente, pela presença de uma condição aguda de saúde com gravidade suficiente para que seja necessária uma intervenção terapêutica ou diagnóstica ou um monitoramento cuidadoso. Inerente a isso, a alta hospitalar é um processo complexo e repleto de desafios ${ }^{3}$. Para tanto, o gerenciamento da alta hospitalar constitui-se em uma ferramenta administrativa, direcionada à promoção de intervenções mais efetivas e planejadas, em uma perspectiva de Integração com outros pontos de atenção à saúde ${ }^{6}$.

Nessa perspectiva, a Continuidade do Cuidado ao paciente após a alta hospitalar evita que o usuário se perca na malha assistencial por desconhecimento dos serviços a que pode recorrer em caso de necessidade. Os déficits nos mecanismos de contrarreferência deixam os usuários e familiares desamparados e inseguros, proporcionando a busca por serviços inadequados, como a procura por Unidades de Pronto Atendimento (UPA), que são destinadas ao atendimento de urgências e emergências ${ }^{4}$.

A busca de informações e ações que sustentam a realização do planejamento de alta no âmbito hospitalar e sua ação responsável constitui o produto final de um percurso que possui conceitos que transversalizam toda a malha assistencial dos níveis de complexidade do Sistema Único de Saúde (SUS) ${ }^{8}$.

As transições entre os serviços de saúde podem ser um período vulnerável, especialmente para usuários com múltiplas comorbidades, regimes de tratamento complicados ou suporte limitado do cuidador. Nesse sentido, práticas que tenham a intenção de aperfeiçoar o gerenciamento da alta hospitalar podem garantir a continuidade da assistência ao paciente e evidenciam um olhar atento para a transição do cuidado ${ }^{9}$.

Nesse cenário de múltiplas necessidades e recursos escassos, enfermeiras e enfermeiros apresentam-se como profissionais estratégicos tanto na gestão de recursos como na coordenação do cuidado, não havendo uma alternativa sem que ele esteja fortemente envolvido. A sua contribuição compreende práticas de integração, transição e de ligação, tendo como objetivo a Continuidade do Cuidado, atendendo não somente a um princípio do SUS, mas também ao direito do cidadão de ser atendido nas suas necessidades de saúde individuais e coletivas ${ }^{4-7}$.

Assim, a Enfermeira de Ligação traduz-se no profissional que possui a função ou atribuição de coordenar a transição de pacientes entre as unidades da rede de atenção, conciliando as necessidades de cuidado e a capacidade da rede em atendê-las com vistas à Continuidade do Cuidado ${ }^{4-6}$.
Portanto, a transição de pacientes com situações complexas do hospital para serviços de cuidados primários depende de uma comunicação eficaz e exige um maior cuidado com todo o contexto envolvido na assistência e alta de cada indivíduo ${ }^{10}$.

Esses, dentre outros aspectos, contribuem com o entendimento de uma dinâmica complexa envolvida no contexto da alta hospitalar e da responsabilidade atribuída aos profissionais, serviços, bem como a todo o Sistema de Saúde. No entanto, muitos conceitos relacionados a este assunto são encontrados na literatura sob várias perspectivas e, portanto, torna-se necessário esclarecêlos a fim de tornar as discussões teóricas mais consistentes e, por consequência, favorecer o cuidado prestado.

Por esse motivo, a fim de promover o conhecimento relacionado ao assunto e de proporcionar uma reflexão a respeito de alguns termos julgados pertinentes ao contexto da gestão da alta hospitalar, este estudo teve como objetivo analisar o conceito Cuidados de Transição de modo a determinar seus atributos críticos, antecedentes e consequentes. Observou-se, por meio de outros estudos ${ }^{4-6}$, uma proximidade entre o conceito pretendido Cuidados de Transição com outros termos, entre esses, destacam-se Enfermeira de Ligação, Continuidade do Cuidado e Integração. Dessa forma, além de clarificar o uso do conceito Cuidados de Transição, pretende-se ratificar ou não essa relação ou sustentar uma diferenciação entre os termos.

\section{MÉTODO}

Trata-se de uma reflexão analítica a respeito do conceito Cuidados de Transição no contexto da gestão da alta hospitalar. Para a sua consecução, realizou-se uma análise teórica mediante o método de análise conceitual de Walker e Avant, que inclui oito passos que podem ocorrer sequencialmente ou de forma simultânea: seleção do conceito; definição do objetivo; identificação do uso do conceito; definição dos atributos; descrição de um caso modelo; descrição de casos adicionais; definição de antecedentes e consequentes; definição de indicadores empíricos ${ }^{1}$.

O primeiro passo consiste na seleção do conceito. $O$ termo Cuidados de Transição reflete parte da experiência profissional e de pesquisa dos autores deste estudo e despertou atenção e preocupação nos últimos anos. O segundo passo corresponde ao objetivo deste estudo.

No terceiro passo, é identificado o uso do conceito por meio de buscas em base de dados e em dicionários para elucidá-lo no campo científico e senso comum ${ }^{1}$. Desta maneira, procedeu-se à busca na literatura entre setembro e dezembro de 2019 na Biblioteca Virtual em Saúde, no MEDLINE/PubMed e na SCOPUS. Foram utilizados termos em inglês e português combinando os descritores identificados no Medical Subject Headings (MeSH), Descritores em Ciências da Saúde (DeCS) e palavras-chave com os operadores booleanos AND e OR.

Optou-se por realizar uma revisão para cada um dos termos considerados pertinentes ao mesmo contexto do conceito abordado neste estudo a fim de promover uma análise teórica e reflexiva que proporcionasse, além do entendimento do conceito, uma compreensão da sua relação com termos que comumente 
são a ele relacionados. Desta forma, utilizou-se como questão norteadora: "Como o conceito Cuidados de Transição relacionase à gestão da alta hospitalar?". O Quadro 1 demonstra as estratégias de busca nas base de dados com as combinações feitas para abranger a busca.

A referida busca obteve um total de 883 artigos. Em seguida, foram aplicados os critérios de inclusão: artigos de pesquisa primária; em português, inglês ou espanhol; textos completos disponíveis on-line na íntegra, sem delimitação de data, nos quais o enfoque fosse cada um dos quatro termos principais (Quadro 1) na gestão da alta hospitalar. Elencaram-se como critérios de exclusão: publicações do tipo editorial; dissertações; teses e resumos. Dois revisores selecionaram os artigos de forma independente e analisaram os títulos e os resumos das publicações identificadas aplicando os critérios de elegibilidade. Os artigos selecionados para a leitura apresentaram, em seu resumo, uma abordagem direta relacionada ao assunto. Foram considerados 77 artigos para a análise, considerando todos os termos elencados. Para fins didáticos e metodológicos, numericamente, consideraram-se apenas os estudos que refletiam o conceito desta investigação, o que resultou em 12 artigos ${ }^{4-6,9,11-18}$. Além destes, 11 registros foram adicionados por meio de busca manual (Figura 1) ${ }^{10,19-28}$.

No quarto passo, são determinados os atributos, ou seja, palavras ou expressões que constituem a essência do conceito e que são pertinentes à análise. $O$ quinto passo consiste em formular um caso modelo, que se traduz em um exemplo de um

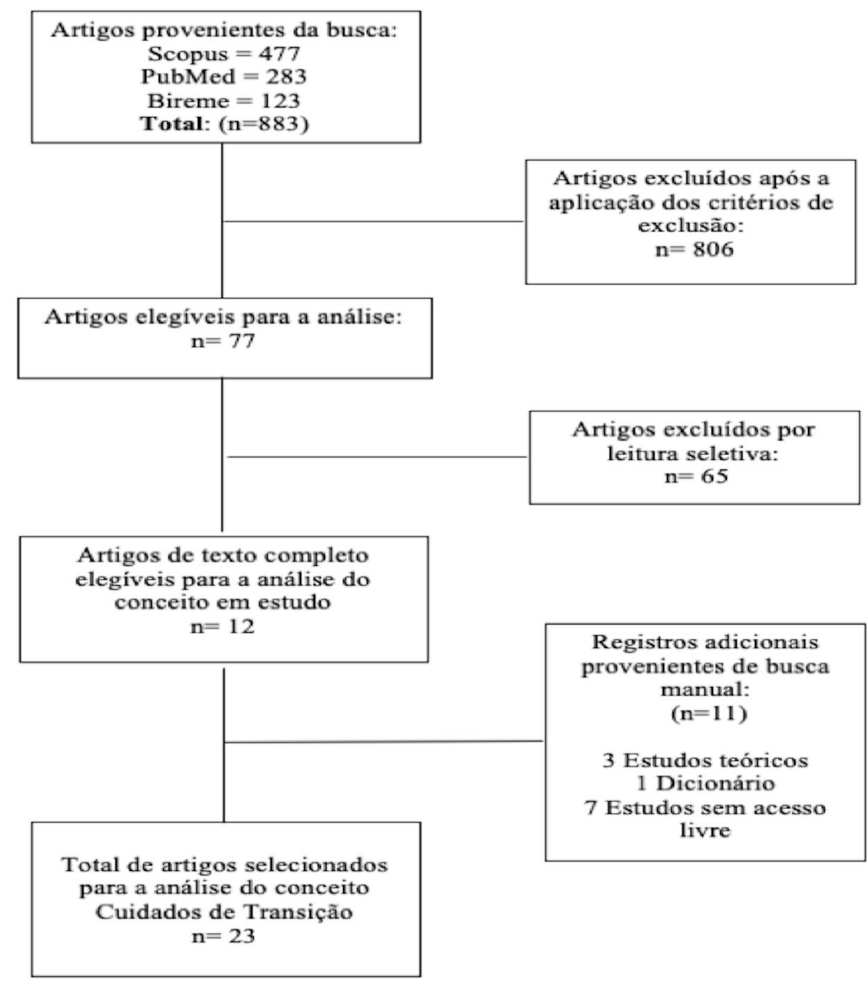

Figura 1. Fluxograma dos estudos selecionados. Curitiba, PR, Brasil, 2021.

Fonte: Elaborada pelos autores (2021).

Quadro 1 - Estratégias de busca. Curitiba, PR, Brasil, 2021.

\begin{tabular}{|c|}
\hline 1) Cuidados de Transição \\
\hline $\begin{array}{l}\text {-"Transitional Care"[MeSH Terms] AND “Patient Discharge"[MeSH Terms] AND “Organization and Administration" [MeSH } \\
\text { Terms] }\end{array}$ \\
\hline $\begin{array}{l}\text {-(tw:("cuidados de transição")) AND (tw: ("alta do paciente")) OR (tw: (“alta hospitalar")) OR (tw:(“gestão de alta")) AND } \\
\text { ("Enfermagem" OR “enfermeiro" OR "enfermeira") }\end{array}$ \\
\hline 2) Continuidade do Cuidado: \\
\hline $\begin{array}{l}\text {-"Continuity of Patient Care" [MeSH Terms] AND "Nurses" [MeSH Terms] AND "Patient Discharge" [MeSH Terms] AND } \\
\text { "Organization and Administration"[MeSH Terms] }\end{array}$ \\
\hline $\begin{array}{l}\text {-"continuidade do cuidado" AND ("alta do paciente" OR "alta hospitalar" OR "gestão de alta") AND (“Enfermagem" OR } \\
\text { "enfermeiro" OR "enfermeira") }\end{array}$ \\
\hline 3) Enfermeira de Ligação: \\
\hline $\begin{array}{l}-" \text { Liaison nurses" [All Fields] AND ("nurses" [MeSH Terms] OR "nurses"[All Fields] OR "nurse"[All Fields])) AND "Patient } \\
\text { Discharge"[MeSH Terms] AND "Organization and Administration" [MeSH Terms] }\end{array}$ \\
\hline -"enfermeiro de ligação" AND "gestão de alta" OR "alta hospitalar" \\
\hline 4) Integração: \\
\hline $\begin{array}{l}\text {-" integrated"[All Fields] OR integration [Text Word] AND "Patient Discharge"[MeSH Terms] AND "Organization and } \\
\text { Administration" [MeSH Terms] AND "integrated"[All Fields] OR integration [Text Word] AND "Int J Integr Care"[Journal] }\end{array}$ \\
\hline $\begin{array}{l}\text {-(tw:("alta hospitalar")) OR (tw:("gestão de alta”)) OR (tw:(“alta do paciente")) AND (tw:(integração)) OR (tw:("assistência } \\
\text { integral à saúde")) OR (tw:("cuidado integral”)) AND (tw:(Enfermagem)) }\end{array}$ \\
\hline
\end{tabular}

Fonte: Elaborado pelos autores (2021) 
caso fictício criado pelos pesquisadores, baseado no contexto real do uso do conceito, abrangendo os atributos identificados. No sexto passo, são identificados casos adicionais. Para isso, foi selecionado um caso contrário ao conceito que colaborou com a construção de características essenciais ${ }^{1}$.

No sétimo passo, ocorre a identificação de antecedentes e consequências do conceito. Trata-se do levantamento de incidentes ou eventos que acontecem antes da ocorrência do conceito e eventos ou situações que surgem depois da presença do conceito. Portanto, foi necessário considerar o contexto social em que o conceito é usado. No oitavo passo, definem-se os referentes empíricos, fenômenos observáveis que confirmam a ocorrência do conceito e permitem um sentido funcional para o mesmo. Muitas vezes, os atributos podem ser similares aos referentes empíricos ${ }^{1} \mathrm{e}$, durante a realização deste estudo, constatou-se que os atributos identificados coincidem com as consequências do conceito e com os referentes empíricos, o que pode ser comum durante o desenvolvimento desta metodologia ${ }^{2}$.

\section{RESULTADOS E DISCUSSÃO}

Os usos do conceito Cuidados de Transição, na perspectiva da área da saúde, e sua definição no dicionário, além dos antecedentes, atributos, consequências e referentes empíricos, são sintetizados no Quadro 2.

\section{Antecedentes}

Os antecedentes cuidados fragmentados $4,12,20$ e reinternação ${ }^{14-16,23,26}$ são citados em vários estudos de diferentes contextos, compreendendo aspectos gerais que instigaram a realização dos estudos. São eventos que motivam não somente os Cuidados de Transição, como também estão relacionados aos termos Integração, Continuidade do Cuidado e Enfermeira de Ligação.

Cuidados fragmentados possuem estreita relação com mudanças de processos fragmentados para processos integrados ${ }^{20}$. Tais mudanças exigem a integração das práticas profissionais de serviços em vários domínios e para diferentes subgrupos, por exemplo, pessoas com diabetes, doenças cardiovasculares etc. ${ }^{13}$ permitindo, assim, que os cuidados realizados em diferentes contextos possam ser continuados.

As reinternações são, de longe, outro importante antecedente a ser analisado como um determinante abrangente. São objeto de estudo de vários autores, que buscam compreender, principalmente, o efeito de práticas que viabilizem uma transição mais segura e a importância de profissionais que as sustentem ${ }^{14-16,23}$.

Consoante a esses estudos, as práticas efetuadas geram associações positivas, com a diminuição das internações e satisfação dos usuários devido a uma melhora em sua qualidade de vida. No entanto, é válido destacar que toda essa discussão deve se dar em paralelo com a implementação de uma via de Cuidados de Transição estruturada que identifique a alta com segurança ${ }^{16}$. Isso, evidentemente, pode ser obtido pelo trabalho capacitado e, frequentemente, citado como Enfermeira de Ligação em muitos cenários e países há várias décadas ${ }^{4-6,23,24}$.

\section{Atributos, consequências e referentes empíricos}

A partir da busca em dicionário, constatou-se que o conceito possui um significado que o aproxima do enfoque principal do estudo ${ }^{19}$. Cuidados de Transição são ações com a finalidade de assegurar a coordenação e a Continuidade dos Cuidados aos indivíduos em transferência entre diferentes serviços ou níveis de complexidade de cuidados. As transições de cuidados são baseadas no nível de cuidados e na disponibilidade de cuidados

Quadro 2. Usos do conceito, antecedentes, atributos, consequências e referentes empíricos.

Curitiba, PR, Brasil, 2021.

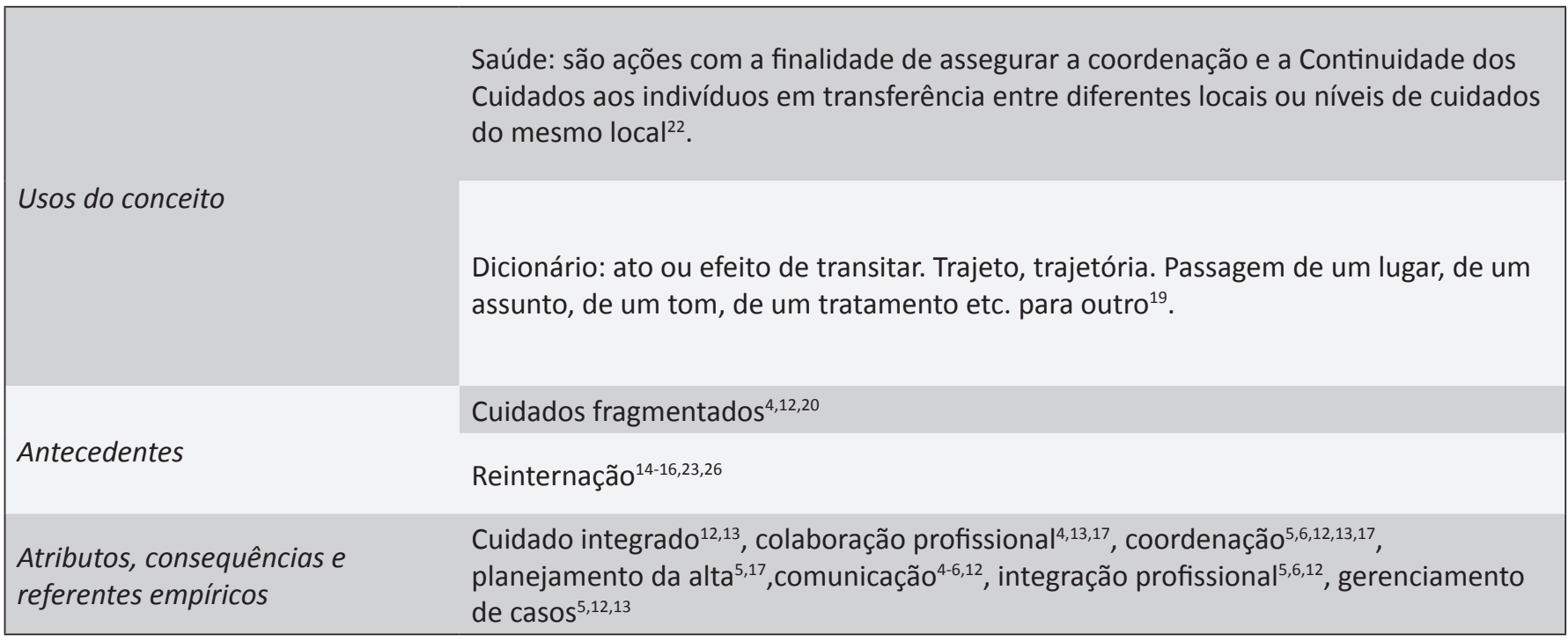

Fonte: Elaborado pelos autores (2020). 
de saúde e abarcam o envio e o recebimento de todos os aspectos da transferência, sendo essencial para os indivíduos com necessidade de cuidados complexos ${ }^{22}$.

No âmbito da transição dos cuidados, a literatura internacional destaca que o adequado planejamento de alta hospitalar com enfoque na Continuidade dos Cuidados resulta em menos readmissões hospitalares ${ }^{26,27}$. Esse contexto é pormenorizado em vários estudos, especialmente em contextos americanos nos quais impera a preocupação em atender aos imperativos da Lei de Cuidados Acessíveis, sancionada em 2010, que, dentre outros aspectos, incentiva não readmissões hospitalares em menos de 30 dias $^{26,27}$.

Em linhas gerais, a Continuidade do Cuidado traduz o que um paciente experimenta ao longo do tempo como coerente e vinculado; este é o desfecho de um bom fluxo de informações, boas habilidades interpessoais e boa coordenação de cuidados. Dessa forma, a Continuidade do Cuidado ocorre quando os elementos separados e discretos do cuidado são conectados e quando esses elementos, que perduram ao longo do tempo, são mantidos e suportados ${ }^{21}$.

Nesse processo, a Enfermeira de Ligação é a profissional que pode possibilitar a redução do tempo de internação e a Continuidade do Cuidado após a alta ${ }^{11}$. É a profissional com capacidade para coordenar e acompanhar todo o percurso dos usuários, devendo, para tanto, ser capaz de realizar a transferência de informações do hospital para a atenção primária, conciliando as necessidades de cuidado com a capacidade da rede em atendê-las em uma perspectiva contínua ${ }^{11}$.

Durante o processo de revisão, constatou-se uma justaposição entre os termos Integração, Continuidade do Cuidado e Enfermeira de Ligação ao conceito Cuidados de Transição, denotando o uso do conceito.

A análise dos principais usos do conceito permitiu destacar os seguintes atributos: cuidado integrado; colaboração profissional, coordenação; planejamento da alta; comunicação; integração profissional; gerenciamento de casos.

O atributo cuidado integrado expressa o cuidado com foco centrado na pessoa e em uma abordagem que prioriza a saúde da população. Isso envolve mudanças em todos os serviços e em toda a rede para garantir que o atendimento seja coordenado de acordo com as necessidades de cada indivíduo, superando a fragmentação na assistência à saúde. Decorrente disso, há a garantia de Integração, que repercute em melhoria no acesso, na qualidade e na Continuidade do Cuidado ${ }^{13}$.

Pode-se presumir um encadeamento consistente do conceito em análise juntamente com os demais termos mencionados de antemão. De maneira similar, o cuidado integrado e de qualidade, ofertado de maneira incondicional ao indivíduo, que resulte em sua alta hospitalar e que anteveja todas as suas necessidades, pode ser considerado o elemento chave para a discussão em curso. Nesse sentido estrito, o conceito Cuidados de Transição, relacionado aos termos Enfermeira de Ligação e Continuidade do Cuidado, possui elementos que asseguram o cuidado integrado e poderiam ser alocados didaticamente em uma condição na qual a Integração perpassa todo o contexto, como o escopo de todo o processo (Figura 2).

No contexto de uma transferência entre serviços de saúde ou destes para o domicílio, deve ser priorizada uma colaboração aprimorada entre os profissionais. Portanto, a prestação de serviços de maneira contínua na transição de um serviço a outro demonstra clara transferência de responsabilidades entre os profissionais ${ }^{17}$. Para tal colaboração profissional, canais de comunicação abertos, tais como prontuários eletrônicos entre as organizações, podem possibilitar a transferência efetiva de informações. Isso torna-se necessário à medida que os profissionais, destacando-se aqui os enfermeiros, possuem entendimento acerca da colaboração existente. Esse reconhecimento é parte primordial de uma colaboração eficaz e facilita o planejamento da alta ${ }^{17}$.

Nesse contexto, introduz-se o atributo coordenação. Profissionais encarregados de coordenar a alta hospitalar são extremamente valiosos, pois podem oferecer apoio aos enfermeiros colaboradores, facilitando a transição segura e suave entre o hospital e a atenção primária ${ }^{17}$. A atuação de coordenadores entre hospital e atenção primária, para apoiar os enfermeiros colaboradores, é extremamente útil. A colaboração e a Continuidade do Cuidado podem ser melhoradas com Enfermeiras de Ligação para coordenar e organizar o planejamento da alta e a comunicação entre o hospital e as organizações de atenção primária ${ }^{17}$.

Outras relações entre o conceito Cuidados de Transição com os termos Enfermeira de Ligação, Continuidade do Cuidado e Integração podem ser apontadas. Por exemplo, as atividades desenvolvidas pela Enfermeira de Ligação para a Continuidade do Cuidado sugerem que a articulação com os serviços minimiza a descontinuidade no cuidado ${ }^{6,23}$ ou, ainda, que os problemas relacionados aos Cuidados de Transição da assistência hospitalar para o domicílio levaram à forte recomendação da introdução do papel de Enfermeira de Ligação ${ }^{6}$. As Enfermeiras de Ligação são essenciais na alta hospitalar e garantem adequado planejamento

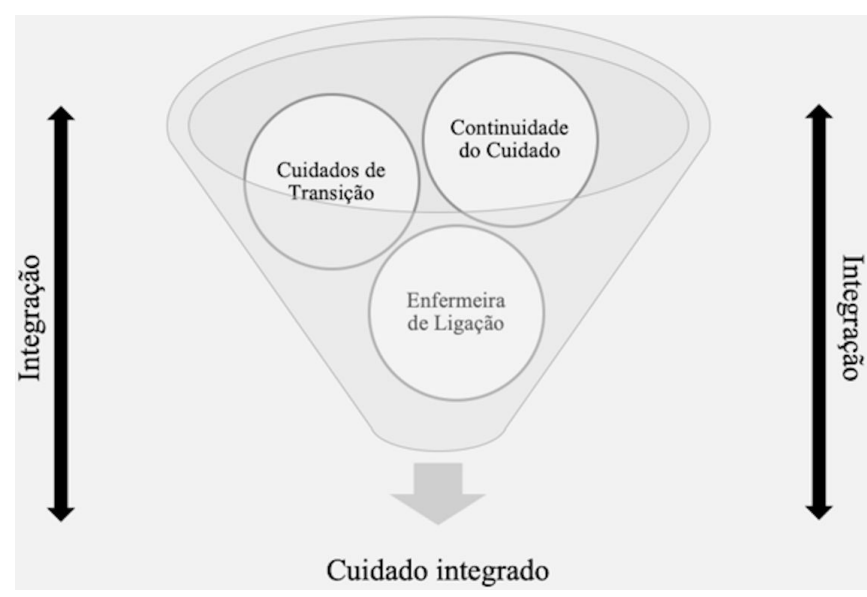

Figura 2. Conceito em análise e sua relação com outros termos. Curitiba, PR, Brasil, 2021.

Fonte: Elaborada pelos autores (2021). 
do cuidado de maneira articulada e coerente com todos os profissionais $^{6}$.

O planejamento eficaz da alta hospitalar tem forte relação com o cuidado integrado e com a transição dos cuidados, repercutindo em melhorias na Integração. Em estudo desenvolvido no Irã, foi apontado que a identificação e o fornecimento de aspectos que favoreçam o planejamento da alta são favoráveis à diminuição das readmissões hospitalares evitáveis, ocasionando a melhoria da qualidade do atendimento ${ }^{18}$. Outros contextos apresentam resultados bem-sucedidos no que se refere à diminuição das taxas de readmissões hospitalares ${ }^{14,15}$. Em Cingapura, um programa de atendimento domiciliar de transição operado por um hospital foi analisado quanto à sua eficácia na redução das hospitalizações. No estudo, 259 pacientes receberam uma avaliação do médico e de um enfermeiro gerente de casos no ambiente doméstico, seguido de um plano de atendimento individualizado que incluía atendimento médico e de Enfermagem, educação do paciente e coordenação do atendimento com especialistas hospitalares e serviços comunitários. Os resultados apontaram, dentre outros aspectos, a redução de atendimentos de emergência, internações, avaliação abrangente das necessidades dos pacientes no ambiente doméstico e a formulação de um plano de atendimento individualizado, otimizando os cuidados pós-alta em situações complexas ${ }^{14}$.

A transição bem-sucedida é o ideal em vários contextos, sobretudo aos relacionados às doenças crônicas. As evidências sugerem que um plano de Cuidados de Transição coordenado pode resultar em uma redução no tempo de internação e nas taxas de readmissão para adultos com necessidades médicas complexas $^{14,15}$. O planejamento da alta tem a finalidade de definir as necessidades dos indivíduos no que se refere à transição de um nível de atendimento para outro, observando-se o contexto que abrange a alta do hospital para a comunidade, e pode ser um eixo central na prestação dos cuidados de Enfermagem. Nesse sentido, inserem-se os Cuidados de Transição como intervenções, usualmente de natureza multidisciplinar, cuja proposta consiste em melhorar os cuidados de saúde por meio da coordenação e Continuidade do Cuidado mais aprimoradas no momento em que os indivíduos transitam entre diferentes serviços ${ }^{14}$.

No estudo desenvolvido em programas de atendimento integrado na Austrália, Canadá, Holanda, Nova Zelândia, Suécia, Reino Unido e Estados Unidos da América ${ }^{13}$, foi possível clarificar os atributos comunicação, integração profissional e gerenciamento de casos. A fim de alinhar os processos de trabalho e, portanto, o tempo e o esforço exigidos por alguns profissionais em detrimento de outros para os cuidados específicos com idosos com quadro de demência, uma iniciativa permitiu que gerentes de caso se encontrassem e discutissem, periodicamente, sobre todos os seus clientes, o que favoreceu a integração profissional ${ }^{13}$. Isso permitiu apreender que a integração do cuidado não tem a ver com descobrir soluções formais e estruturais para tudo ${ }^{14}$. A confiança e as boas relações de trabalho parecem ter desempenhado um papel importante na superação da fragmentação que desafia os processos de trabalho estabelecidos ${ }^{13}$. Enquanto a integração de estruturas e processos funcionais desempenharam papéis facilitadores indispensáveis na prestação de serviços, as pequenas equipes e a pouca hierarquia permitiram a comunicação entre todos, considerando ainda a situação do usuário como ponto de partida ${ }^{13}$.

De várias maneiras análogas, esses atributos caminham juntos e possuem elementos técnicos e práticos para o alcance e vislumbre do conceito desta investigação ${ }^{5,6,10}$.

Como parte da metodologia proposta, o Quadro 3 exemplifica o conceito Cuidados de Transição e estabelece a conexão com os termos Enfermeira de Ligação, Continuidade do Cuidado e Integração por meio de um contexto fictício no qual estão presentes os atributos: cuidado integrado; colaboração profissional; coordenação; planejamento da alta; comunicação; integração profissional e gerenciamento de casos.

Percebe-se, no evento descrito, que a preocupação com uma alta hospitalar de qualidade pode prevenir eventuais reinternações, promovendo, dessa forma, um cuidado contínuo. Para tanto, são necessários profissionais engajados em garantir

Quadro 3. Caso modelo. Curitiba, PR, Brasil, 2021.

A.C.M, mulher, operadora de caixa, 51 anos, casada. Internada em um hospital escola, admitida após Infarto Agudo do
Miocárdio (IAM), submetida a cateterismo cardíaco de emergência seguido de angioplastia para tratamento. História de
hipertensão, diabetes e hipercolesteronemia. Estava em consulta com a enfermeira da Unidade de Saúde do seu bairro quando
apresentou sinais pouco específicos de IAM (dores intermitentes na mandíbula e escápula e dor leve no estômago). É atendida
prontamente pela enfermeira e pelo médico da unidade, que identificam um possível quadro mais complexo e acionam o
Serviço de Atendimento Móvel de Urgência (SAMU). Antes de encaminhar para o hospital, médico e enfermeira realizam
algumas observações do estado da paciente, sinais vitais e tomam algumas medidas no momento dos sintomas. Seguem-se
os cuidados na Unidade de Terapia Intensiva (UTI). No dia seguinte, a enfermeira da UTI realiza contato com a enfermeira
da unidade de saúde buscando informações adicionais da paciente. O contato é realizado e a enfermeira do hospital obtém
informações adicionais que a auxiliam a elaborar o planejamento da alta. Em meio a isso, no hospital, alguns profissionais
reúnem-se duas vezes ao mês para a discussão de alguns casos e, em um uma dessas ocasiões, o caso de A.C.M foi discutido a
fim de traçar seu melhor plano terapêutico, visto que, durante a internação, voltou a apresentar sintomas que precisariam ser
rediscutidos para traçar nova conduta. No momento da alta hospitalar, a enfermeira do hospital entrou em contato novamente
com a unidade de saúde e relatou, à enfermeira da unidade de saúde, as informações pertinentes à internação de A.C.M.

Fonte: Elaborado pelos autores (2021). 
Quadro 4. Caso contrário. Curitiba, PR, Brasil, 2021. Homem afro-americano de 73 anos, o Sr. W., internado em um grande hospital universitário urbano com glicemia de 560 mg/dL,
desidratação e confusão. Ele melhorou com insulina e hidratação e conseguiu dar, à equipe do hospital, o número de telefone de
sua esposa. Após uma discussão entre a equipe do hospital e sua esposa, o Sr. W. foi enviado para casa com um encaminhamento
para serviços de atendimento domiciliar e o mesmo regime diabético que ele tinha antes da visita ao hospital. Seu médico
de cuidados primários não foi contatado. Cinco dias depois, o Sr. W. voltou ao Departamento de Polícia depois que a polícia o
encontrou vagando pelas ruas. Testes adicionais revelaram demência vascular moderada, hiperglicemia e desidratação. Além
disso, sua esposa é deficiente, frequenta uma creche e não pode ajudá-lo com seu regime ou dieta diabética. Tais aspectos
apontados requerem várias visitas e internações de emergência. As preocupações deste paciente apontam claramente para
a necessidade de melhores transições ${ }^{28}$.

Fonte: Burke ${ }^{28}$

esse processo em um contexto em que vários serviços atuam para garantir a assistência de qualidade e integrada.

É oportuno ressaltar que a discussão em torno de um cuidado integral, contínuo, coordenado e que satisfaça as necessidades de saúde dos indivíduos compreende ainda a perspectiva da referência e da contrarreferência e que uma adequada organização para os encaminhamentos de média e alta complexidade pode favorecer a transição do cuidado.

Como exemplo de um caso adicional, é apresentado, no Quadro 4, um caso contrário ${ }^{28}$.

Neste caso, não é possível identificar os atributos relacionados ao conceito em análise, mas informações opostas, contribuindo para a compreensão das características dos conceitos.

\section{CONCLUSÃO E IMPLICAÇÕES PARA A PRÁTICA}

Foi oportuno analisar o conceito Cuidados de Transição em conjunto com os termos Enfermeira de Ligação, Continuidade do Cuidado e Integração, pois todos estão entrelaçados no contexto da alta hospitalar. A caracterização do conceito foi possível mediante os atributos discutidos, ou seja, cuidado integrado, colaboração profissional, coordenação, planejamento da alta, comunicação, integração profissional e gerenciamento de casos perfazem aspectos importantes dos conceitos, possibilitando definir um sentido.

A modo de desfecho, conceitua-se, a partir deste estudo, que os Cuidados de Transição são práticas coordenadas, comprovadamente eficazes para garantir segurança e Continuidade dos Cuidados durante a transferência do usuário na alta hospitalar na tentativa de garantir qualidade de vida e, por conseguinte, evitar reinternações hospitalares. Nesse contexto, os profissionais denominados Enfermeiras de Ligação são potenciais protagonistas para estar à frente dessas práticas, contribuindo com a gestão da alta hospitalar e com o processo de Integração.

As especificidades relacionadas ao conceito em estudo poderão favorecer a sua compreensão de maneira precisa e a construção de conhecimentos que repercutam em um cuidado coordenado e contínuo. Enfatiza-se a afinidade entre o conceito e os termos descritos, especialmente no que diz respeito à Enfermeira de Ligação, no contexto da gestão da alta hospitalar.
Como limitação, o estudo não contemplou todos os contextos inerentes à rede de atenção.

E, para tanto, como possibilidade de novos estudos, pesquisas realizadas em contextos diferentes originariam outros atributos e definições. Além disso, é importante mencionar que a metodologia foi oportuna, possibilitando o alcance do objetivo. Contudo, os resultados obtidos a partir de outras técnicas de análise de conceito poderão agregar mais conhecimento.

\section{FINANCIAMENTO}

Projeto financiado pelo Edital no 09/2018, do Conselho Nacional de Desenvolvimento Científico e Tecnológico (CNPq), concedido à Profa. Dra. Elizabeth Bernardino. Processo no 307642/2018-6. Título do projeto de pesquisa: "Da admissão à alta: conceitos e estratégias para ações de continuidade do cuidado em Enfermagem em hospital de ensino".

\section{CONTRIBUIÇÕES DOS AUTORES}

Desenho do estudo de reflexão Elizabeth Bernardino. Solange Meira de Sousa. Jaqueline Dias do Nascimento.

Levantamento de fontes/informações, Elizabeth Bernardino. Solange Meira de Sousa. Maria Ribeiro Lacerda.

Análise. Elizabeth Bernardino. Solange Meira de Sousa

Luciana Schleder Gonçalves.

Interpretação dos resultados. Elizabeth Bernardino. Solange

Meira de Sousa. Jaqueline Dias do Nascimento. Maria Ribeiro Lacerda. Danelia Gómez Torres.

Redação e revisão crítica do manuscrito. Elizabeth Bernardino. Solange Meira de Sousa. Jaqueline Dias do Nascimento. Maria Ribeiro Lacerda. Danelia Gómez Torres. Luciana Schleder Gonçalves.

Aprovação da versão final do artigo. Elizabeth Bernardino. Solange Meira de Sousa. Jaqueline Dias do Nascimento. Maria Ribeiro Lacerda. Danelia Gómez Torres. Luciana Schleder Gonçalves.

Responsabilidade por todos os aspectos do conteúdo e a integridade do artigo publicado. Elizabeth Bernardino. Solange Meira de Sousa. Jaqueline Dias do Nascimento. Maria Ribeiro Lacerda. Danelia Gómez Torres. Luciana Schleder Gonçalves 


\section{EDITOR CIENTÍFICO}

Ivone Evangelista Cabral (1)

\section{EDITOR ASSOCIADO}

\author{
Rodrigo Nogueira da Silva (1)
}

\section{REFERÊNCIAS}

1. Walker LO, Avant KC. Strategies for theory construction in nursing. 6th ed. Nova York, NY (USA): Editora Person; 2019.

2. Fernandes M, da Nóbrega MM, Garcia TR, Macêdo-Costa KN. Conceptual analysis: methodological considerations. Rev Bras Enferm. 2011;64(6):1150-6. http://dx.doi.org/10.1590/S0034-71672011000600024. PMid:22664616.

3. Kuo YF, Goodwin JS. Association of hospitalist care with medical utilization after discharge: evidence of cost shift from a cohort study. Ann Intern Med. 2011;155(3):152-9. http://dx.doi.org/10.7326/00034819-155-3-201108020-00005. PMid:21810708.

4. Ribas EN, Bernardino E, Larocca LM, Poli P No, Aued GK, Silva CPCD. Nurse liaison: a strategy for counter-referral. Rev Bras Enferm. 2018;71(Suppl 1):546-53. http://dx.doi.org/10.1590/0034-7167-20170490. PMid:29562010.

5. Costa MFBNAD, Andrade SR, Soares CF, Pérez EIB, Tomás SC Bernardino E. The continuity of hospital nursing care for Primary Health Care in Spain. Rev Esc Enferm USP. 2019;53:e03477. http://dx.doi. org/10.1590/s1980-220x2018017803477. PMid:31291396.

6. Aued GK, Bernardino E, Lapierre J, Dallaire C. Atividades das enfermeiras de ligação na alta hospitalar: uma estratégia para a continuidade do cuidado. Rev Lat Am Enfermagem. 2019;27:e3162. http://dx.doi. org/10.1590/1518-8345.3069.3162. PMid:31432917.

7. Sousa SM, Bernardino E, Crozeta K, Peres AM, Lacerda MR. Integrality of care: challenges for the nurse practice. Rev Bras Enferm. 2017;70(3):50410. http://dx.doi.org/10.1590/0034-7167-2016-0380. PMid:28562797.

8. Portaria $n^{\circ} 3390$ de 30 dezembro de 2013 (BR). Institui a Política Nacional de Atenção Hospitalar (PNHOSP) no âmbito do Sistema Único de Saúde (SUS), estabelecendo- se as diretrizes para a organização do componente hospitalar da Rede de Atenção à Saúde (RAS). Diário Oficial da União [periódico na internet], Brasília (DF), 2013 [citado 2020 out 15]. Disponível em: https://bvsms.saude.gov.br/bvs/saudelegis/ gm/2013/prt3390_30_12_2013.html

9. Reidt SL, Holtan HS, Larson TA, Thompson B, Kerzner LJ, Salvatore $\mathrm{TM}$ et al. Interprofessional collaboration to improve discharge from skilled nursing facility to home: preliminary data on postdischarge hospitalizations and emergency department visits. J Am Geriatr Soc. 2016;64(9):1895-9. http://dx.doi.org/10.1111/jgs.14258. PMid:27385197.

10. Rattray NA, Sico JJ, Cox LM, Russ AL, Matthias MS, Frankel RM. Crossing the communication chasm: challenges and opportunities in transitions of care from the hospital to the primary care clinic. Jt Comm J Qual Patient Saf. 2017;43(3):127-37. http://dx.doi.org/10.1016/j. jcjq.2016.11.007. PMid:28334591.

11. Hesselink G, Zegers M, Vernooij-Dassen M, Barach P, Kalkman C, Flink $M$ et al. Improving patient discharge and reducing hospital readmissions by using intervention mapping. BMC Health Serv Res. 2014;14(1):389. http://dx.doi.org/10.1186/1472-6963-14-389. PMid:25218406.

12. Fitzpatrick SJ, Perkins D, Handley T, Brown D, Luland T, Corvan E. Coordinating mental and physical health care in rural australia: an integrated model for primary care settings. Int J Integr Care. 2018;18(2):19. http://dx.doi.org/10.5334/ijic.3943. PMid:30127703.
13. Wodchis WP, Dixon A, Anderson GM, Goodwin N. Integrating care for older people with complex needs: key insights and lessons from a seven-country cross-case analysis. Int J Integr Care. 2015;15(6):e021. https://doi.org/10.5334/ijic.2249.

14. Low LL, Vasanwala FF, Ng LB, Chen C, Lee KH, Tan SY. Effectiveness of a transitional home care program in reducing acute hospital utilization: a quasi-experimental study. BMC Health Serv Res. 2015;15:100. https:// doi.org/10.1186/s12913-015-0750-2.

15. Diplock G, Ward J, Stewart S, Scuffham P, Stewart P, Reeve C et al. The Alice Springs Hospital Readmission Prevention Project (ASHRAPP): a randomised control trial. BMC Health Serv Res. 2017;17(1):153. http:// dx.doi.org/10.1186/s12913-017-2077-7. PMid:28219383.

16. Soto GE, Huenefeldt EA, Hengst MN, Reimer AJ, Samuel SK, Samuel SK et al. Implementation and impact analysis of a transitional care pathway for patients presenting to the emergency department with cardiac-related complaints. BMC Health Serv Res. 2018;18:672. https:// doi.org/10.1186/s12913-018-3482-2.

17. Lemetti T, Voutilainen $P$, Stolt M, Eloranta S, Suhonen R. An enquiry into nurse-to-nurse collaboration within the older people care chain as part of the integrated care: a qualitative study. Int J Integr Care. 2017;17(1):5. http://dx.doi.org/10.5334/ijic.2418. PMid:29042847.

18. Gholizadeh M, Janati A, Delgoshaei B, Gorji HA, Tourani S. Implementation requirements for Patient Discharge Planning in Health System: a qualitative study in Iran. Ethiop J Health Sci. 2018;28(2):157-68. http:// dx.doi.org/10.4314/ejhs.v28i2.7. PMid:29983513.

19. Ferreira ABH. Dicionário Aurélio da Língua Portuguesa. 5. ed. Curitiba: Positivo; 2010.

20. Nuño-Solinís R. Revisiting organisational learning in integrated care. Int J IntegrCare.2017;17(4):4. http://dx.doi.org/10.5334/ijic.3047.PMid:28970762.

21. Reid RJ, Haggerty J, Mckendry R. Defusing the confusion: concepts and measures of continuity of health care. [Internet]. Canadian Health Services Research Foundation; 2002 [citado 2020 out 15]. Disponíve em: https://www.researchgate.net/publication/245856177_Defusing the Confusion_Concepts_and_Measures_of_Continuity_of_Health_Care

22. Coleman EA, Boult CE, American Geriatrics Society Health Care Systems Committee. Improving the quality of transitional care for persons with complex care needs. J Am Geriatr Soc. 2003;51(4):556-7. http://dx.doi. org/10.1046/j.1532-5415.2003.51186.x. PMid:12657079.

23. Vat M, Common C, Laizner AM, Borduas C, Maheu C. Reasons for returning to the emergency department following discharge from an internal medicine unit: perspectives of patients and the liaison nurse clinician. J Clin Nurs. 2015;24(23-24):3605-14. http://dx.doi.org/10.1111/ jocn.13011. PMid:26467172.

24. Arts SE, Francke AL, Hutten JB. Liaison nursing for stroke patients: results of a Dutch evaluation study. J Adv Nurs. 2000;32(2):292-300. http://dx.doi.org/10.1046/j.1365-2648.2000.01476.x. PMid:10964175.

25. Glimmerveen L, Nies H, Ybema S. Citizens as active participants in integrated care: challenging the Field's dominant paradigms. Int J Integr Care. 2019;19(1):6. http://dx.doi.org/10.5334/ijic.4202. PMid:30881264.

26. Cloonan P, Wood J, Riley JB. Reducing 30-day readmissions: health literacy strategies. J Nurs Adm. 2013;43(7-8):382-7. http://dx.doi. org/10.1097/NNA.0b013e31829d6082. PMid:23892303.

27. Kind AJH, Jensen L, Barczi S, Bridges A, Kordahl R, Smith MA et al Low-cost transitional care with nurse managers making mostly phone contact with patients cut rehospitalization at a VA hospital. Health Aff (Millwood). 2012;31(12):2659-68. http://dx.doi.org/10.1377/ hlthaff.2012.0366. PMid:23213150.

28. Burke M. Transitional care. J Gerontol Nurs. 2009;35(12):3-4. http:// dx.doi.org/10.3928/00989134-20091103-05. PMid:20000268. 